\title{
Saccharomyces cerevisiae to Mutants Resistant $\beta$-L-Aspartylhydroxamate
}

\author{
By GARY E. JONES* \\ Department of Botany and Plant Sciences, Cell Interaction Group, University of California, \\ Riverside, CA 92521, USA
}

(Received 17 October 1984; revised 22 March 1985)

\begin{abstract}
Yeast strains resistant to the $\mathrm{L}$-asparagine analogue $\beta$-L-aspartylhydroxamate have been shown to have mutations in any of at least three unlinked genes. Mutation in one of these genes, $a h r l$, is dominantly expressed and affects a function that involves nitrogen metabolism in the cells, including the exclusion of amino acids at the level of transport. The general amino acid permease is rendered sensitive to ammonium ion in strains carrying the dominantly expressed mutations in $a h r l$, but other functions related to nitrogen metabolism probably are involved as well.
\end{abstract}

\section{INTRODUCTION}

The amino acid analogue $\beta$-aspartylhydroxamate (BAH), which has a hydroxylamine group in place of the amide group of asparagine, has been used in the study of asparagine metabolism in several organisms. Gantt et al. (1980) and Andrulis \& Siminovitch (1982) isolated cultured animal cells resistant to growth inhibition by L-BAH. They proved to be overproducers of Lasparagine because they possessed elevated L-asparagine synthetase activity. Kinghorn \& Pateman (1977) described mutants of Aspergillus nidulans that are resistant to BAH, and Drainas et al. (1977) and Arst \& Bailey (1980) selected strains of $A$. nidulans that were resistant to growth inhibition by DL-BAH, and showed that they lacked asparaginases that normally degrade Lasparagine to L-aspartate and ammonium ion. In wild-type cells of this species, the asparaginase releases the hydroxylamine moiety from the analogue, which then kills the cells. Mutants lacking the asparaginase do not release the toxic moiety and are resistant to the analogue. Recently, Kim \& Roon (1984) used a clever selection scheme involving D-BAH to specifically select mutants of the yeast Saccharomyces cerevisiae that lack activity of asparaginase II, a highly regulated extracellular deamidase that uses either D- or L-asparagine as a substrate.

In this paper is presented a genetic and phenotypic characterization of mutants of $S$. cerevisiae that are resistant to L-BAH, focusing mainly on a class in which resistance is dominantly expressed. The biochemical cause of resistance in these strains has not been identified, but neither overproduction of L-asparagine nor loss of asparaginase activity seems to be involved. These mutations appear to pleiotropically affect the regulation of nitrogen metabolism in this species.

Nitrogen metabolism is not an area with which our laboratory deals in depth. The findings presented here are to alert others to the possible value of using L-BAH in such investigations, and to suggest that a detailed biochemical study of a spectrum of mutants resistant to this analogue might provide significant new insight in these fields.

\section{METHODS}

Strains. S. cerevisiae strain S288C ( $M A T \alpha S U C 2$ mal gal2 CUPl mel), our standard wild-type strain, originated from the Yeast Genetics Stock Center, University of California, Berkeley, CA, USA. Strains PL1204 and PL1420, which were derived from the wild-type strain $\Sigma 1278$ b (Lasko \& Brandriss, 1981), were kindly supplied by Dr Marjorie C. Brandriss, University of Medicine and Dentistry of New Jersey, USA. Mutations carried by PL1204

Abbreviations: BAH, $\beta$-L-aspartylhydroxamate (L-aspartic acid $\beta$-hydroxamate); GAP, general amino acid permease; GGH, $\gamma$-L-glutamylhydroxamate (L-glutamic acid $\gamma$-hydroxamate). 
and PL1420 (aap-82 and gapl, respectively) were transferred to strains that are nearly isogenic with other strains used in the study by repeated backcrosses to $\mathrm{S} 288 \mathrm{C}$ or one of its derivatives.

Media. Synthetic medium (SD) and enriched medium (YPD) have been described previously (Sherman et al., 1971). The nitrogen source in SD medium is stated in the appropriate places in the text. SD20 and SD75 are synthetic media containing $20 \mathrm{~mm}$ - or $75 \mathrm{~mm}$-ammonium ion, respectively (as ammonium sulphate).

Growth of cells. Cells were grown at $28^{\circ} \mathrm{C}$ on gyratory shakers at 180 r.p.m. Culture titres were determined by measuring $\mathrm{OD}_{520}$ in $22 \mathrm{~mm}$ culture tubes or by counting cells in a haemocytometer. The initial $\mathrm{pH}$ of all media was adjusted to $4 \cdot 2$.

Enzyme activities. L-Asparaginase I was assayed in permeabilized cells as described by Jones \& Mortimer (1973), except that toluene was used in place of benzene, and assays were done at $28^{\circ} \mathrm{C}$. Asparaginase II activity was assayed by a modification of the method of Dunlop \& Roon (1975) that has been described by Pauling \& Jones (1980). D-Asparagine (10 mM) was used as the substrate. Ammonium ion concentrations were determined spectrophotometrically by coupling with glutamate dehydrogenase (Pauling \& Jones, 1980). L-Asparagine synthetase activity was assayed by the method of Ramos \& Wiame (1979).

Amino acid transport. Cells were grown overnight in the appropriate medium, diluted to approximately $2 \times 10^{6}$ cells $\mathrm{ml}^{-1}$ (haploids) or $1 \times 10^{6}$ cells ml-1 (diploids), and allowed to grow to approximately $7 \times 10^{6} \mathrm{cells}^{-1} \mathrm{or}^{-1}$ $3.5 \times 10^{6}$ cells $\mathrm{ml}^{-1}$, respectively (mid-exponential phase). Cells were collected by low speed centrifugation, washed with SD medium containing no nitrogen source, suspended in SD without nitrogen, and pipetted into small beakers on a gyratory platform in a water bath at $28^{\circ} \mathrm{C}$. After about $10 \mathrm{~min}$, a concentrated solution of the appropriate ${ }^{14} \mathrm{C}$-labelled substrate was added to give a final concentration of $0.10 \mathrm{~mm}$ at a specific radioactivity of 500-2000 d.p.m. nmol ${ }^{-1}$. Samples of $1.0 \mathrm{ml}$ were collected at intervals in tubes at $0^{\circ} \mathrm{C}$ containing excess unlabelled substrate (usually $5 \mathrm{~mm}$ ). Cells were collected on membrane filters and washed three times with cold, concentrated, unlabelled substrate in distilled water. Filters were put into scintillation vials and dried, and radioactivity accumulated by the cells was determined by liquid scintillation counting at a counting efficiency of about $80 \%$. Under these conditions, the $\mathrm{pH}$ during transport was approximately $4 \cdot 2$, and uptake of label was linear with time.

Protein concentration. Protein content of samples was determined by the Lowry method as described previously (Pauling \& Jones, 1980), or by the method of Bradford (1976).

Genetic methods. Genetic analyses were done as described previously (Mortimer \& Hawthorne, 1969). Diploid cells were selected from mating mixtures by micromanipulation, and diploidy was confirmed by sporulation.

Chemicals. All chemicals were from Sigma. Radioisotopes were from ICN except radiolabelled L-citrulline and L-asparagine, which were from New England Nuclear.

\section{RESULTS AND DISCUSSION}

\section{Responses to $\mathrm{L}-B A H$}

Cells of strains S288C and XE227-20A (MAT $\alpha$ asp1-12 asp2 asp3) were cultured in SD75 medium containing various concentrations of L-BAH (hereafter abbreviated BAH). Strain XE227-20A lacks both L-asparaginase I, the internal yeast asparaginase (Jones \& Mortimer, 1970), and asparaginase II, the external asparaginase described above. (The asp1-12 mutation confers loss of L-asparaginase I activity; either the asp 2 or the asp 3 mutation results in loss of asparaginase II.) The analogue inhibited growth in a concentration dependent fashion (cf. Fig. 1 and Fig. 2). At the highest concentration of BAH tested, cells of both strains underwent approximately 1.5 divisions before growth ceased. In a separate experiment, $\mathrm{S} 288 \mathrm{C}$ and strains lacking one or both the asparaginases were cultured in SD75 containing $0.25 \mathrm{mM}-\mathrm{BAH}$, a concentration that inhibits growth intermediately. The degree of inhibition of all strains was similar. These results show that lack of asparaginase activity alone does not lead to resistance to this asparagine analogue under the conditions used.

\section{Isolation of BAH-resistant mutants}

When cultures of sensitive cells were incubated for extended periods in medium containing fully inhibitory concentrations of $\mathrm{BAH}$, growth often resumed after $50-70 \mathrm{~h}$ and continued at a nearly normal rate. One possible explanation for this observation was that resistant cells occurring spontaneously had taken over the cultures. Subsequent testing showed that this was the case. To isolate BAH-resistant mutants of independent origin, clonal populations of cells of strains S288C or XE227-20A were seeded at low density into SD75 medium containing $1.0 \mathrm{~mm}$ $\mathrm{BAH}$. When resistant cells had grown in the cultures, samples were diluted and plated onto YPD medium containing no analogue. Single colonies were picked to originate clonal populations of 


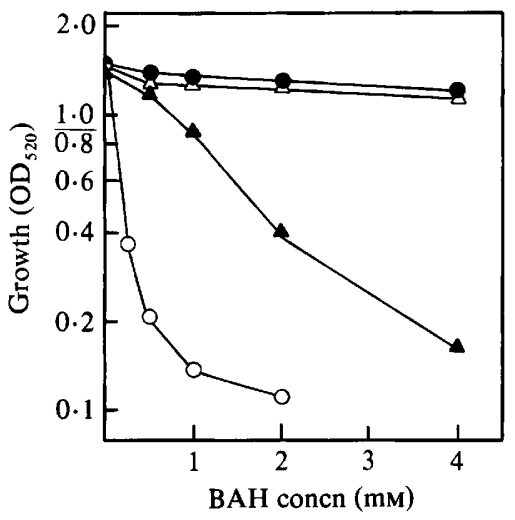

Fig. 1

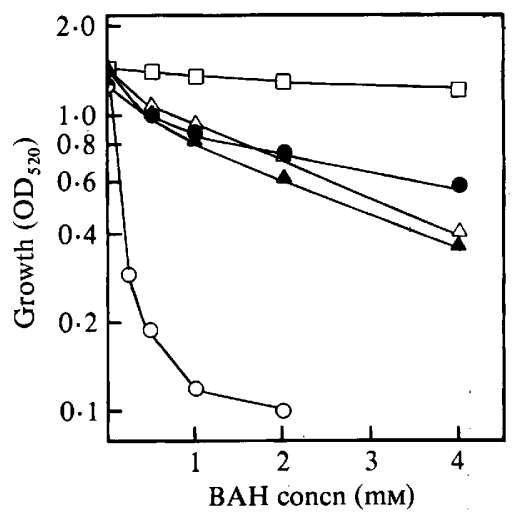

Fig. 2

Fig. 1. Growth of $S$. cerevisiae $\mathbf{S 2 8 8 C}$ and BAH-resistant strains derived from it in SD75 medium containing the indicated concentrations of $\mathrm{BAH}$. Cultures were initiated at low $\mathrm{OD}_{520}$ (approximately 0.03 , equivalent to about $1 \times 10^{6}$ cells $\mathrm{mi}^{-1}$ ), incubated as described in Methods, and the $\mathrm{OD}_{520}$ was measured after $20 \mathrm{~h}$. At this time, control cultures containing no BAH were in late transition phase (about $5 \times 10^{7}$ cells $\mathrm{ml}^{-1}$ ). Under these conditions, an OD of 0.1 corresponds to about $3 \times 10^{6}$ cells $\mathrm{ml}^{-1}$. Points represent averages of duplicate cultures, which did not differ by more than $10 \%$. O, S288C; $\triangle$, CAHR3; O, CAHR7; $\triangle$, CAHR9.

Fig. 2. Growth of XE227-20A and BAH-resistant derivatives in SD75 medium containing BAH. Conditions were as described in Fig. 1. O, XE227-20A; $\triangle$ AAHR1; , AAHR3; $\square$, AAHR5; $\Delta_{\text {, }}$ AAHR8.

the independent mutants. In this way, three resistant mutants (CAHR3, CAHR7, and CAHR9) were derived from S288C and four (AAHR1, AAHR3, AAHR5, and AAHR8) from XE227$20 \mathrm{~A}$. The responses of these strains to graded concentrations of BAH are shown in Figs 1 and 2.

\section{Genetics of resistance to $B A H$}

To test whether resistance was dominant or recessive, each of the mutants was crossed with strain XE59-8B (MATa wild-type equivalent to S288C), and the growth of the diploid cells in medium containing $0.5 \mathrm{~mm}-\mathrm{BAH}$ was assayed. Cells heterozygous for mutations carried by strains CAHR9 or AAHR5 grew nearly as well in medium containing BAH as wild-type diploid cells in medium without the analogue (Fig. 3). Even at a BAH concentration of $1.0 \mathrm{~mm}$, growth of these heterozygotes was only slightly affected. Thus, these mutations were dominantly expressed.

Heterozygotes carrying mutations that arose in strains CAHR3, CAHR7, AAHR1, AAHR3 and AAHR8 were inhibited in growth by BAH to about the same extent as wild-type cells, and thus these mutations were recessively expressed.

Diploid cells heterozygous for each of the seven presumptive mutations were sporulated, dissected, and analysed for segregation of resistance. In all cases except one, resistance segregated in the 2 sensitive : 2 resistant manner expected of a trait determined by two alleles of a single gene. Resistance in strains carrying mutation(s) that arose in AAHR5 segregated as if two genes were involved, and expression of resistance appeared to involve a complex interaction between these genes.

\section{Complementation tests}

Diploid cells carrying the recessively expressed mutations in all pairwise combinations were replica plated onto SD75 medium containing $1.0 \mathrm{~mm}-\mathrm{BAH}$ and onto medium without analogue. The plates were scored for growth one and two days later. These tests showed that mutations from CAHR3 and CAHR7 were in one cistron, those from AAHR1 and AAHR3 represented another cistron, and that from AAHR8 was in yet a third one. In all cases, complementation (lack of growth on BAH medium) was strong and was not typical of intragenic 


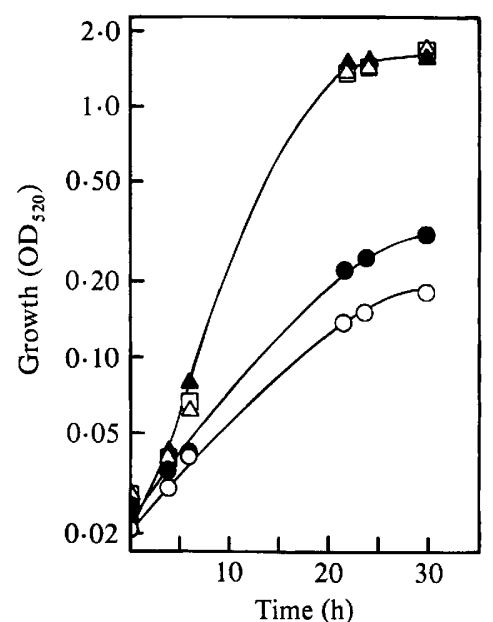

Fig. 3

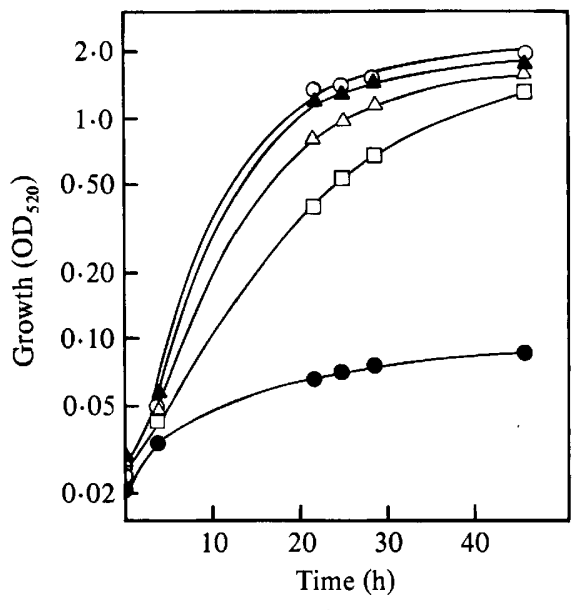

Fig. 4

Fig. 3. Growth of diploid strains in SD75 medium containing 0-5 mM-BAH. O, XE521 (wild-type); $\triangle$, XE597 (+/AHRI-9); $\square$, XE598 (AHRI-9/AHR1-9); $\bigcirc$, XE596 (gapl/gapl); $A$, XE521 (wild-type) in medium containing no BAH. Similar results were obtained with strains derived from AAHR5. The mutant allele $A H R I-9$ arose originally in BAH-resistant strain CAHR9.

Fig. 4. Growth of strains of haploid cells derived from S288C containing mutations engendering dominantly expressed resistance to $\mathrm{BAH}$. The concentration of $\mathrm{BAH}$ was $1 \mathrm{mM} . \mathrm{O}, \mathrm{S} 288 \mathrm{C}$ in medium containing no BAH;, $\mathrm{S} 288 \mathrm{C}$ in medium with BAH; $\triangle$, CAHR9, CAHR10; $\Delta$, CAHR11, CAHR12, CAHR14; $\square$, CAHR13. (Strains CAHR9-CAHR14 all in medium containing $\mathrm{BAH}$.) Where only a single symbol is used for more than one strain, responses of those strains were indistinguishable.

complementation. Recombinational analyses confirmed the results of the complementation tests and showed that none of the three cistrons were closely linked (results not shown).

At this point, it became clear that resistance to BAH might have several aetiologies and probably involves the products of several genes. In the remainder of this paper are described the effects of dominantly expressed mutations that arose in S288C or its diploid equivalent.

\section{Isolation of resistant diploid cells}

Diploid cells of strain XE521, the product of mating between wild-type strains S288C and XE59-8B, were incubated at $28^{\circ} \mathrm{C}$ in SD75 medium containing $1.0 \mathrm{mM}-\mathrm{BAH}$ until presumptive resistant cells appeared. Five independent resistant strains were selected and given identifying numbers CAHR10 through CAHR14. Each diploid was sporulated and the segregation of resistance ascertained. In all five cases, resistance and sensitivity segregated $2: 2$ in haploid progeny, showing that each resistant diploid was heterozygous for two alleles of a gene, one of which engenders dominant resistance to the analogue. Subsequent crosses showed that four of these mutations and the one from CAHR9 segregated as if they were in a single gene, although mutation in two closely linked genes could not be ruled out entirely. This gene was provisionally designated $A H R 1$, the resistance alleles being $A H R 1-9$ through $A H R 1-12$, and $A H R 1-14$, corresponding to their origination in strains CAHR9 through CAHR12, and CAHR14, respectively. The original sensitivity allele in strain S288C was named ahrl-1. The dominant mutation carried by strain CAHR 13 assorted from the others in some asci, but we could not unambiguously assign it to another locus in these initial studies. Although it was included in further studies because phenotypically it was similar to the other mutations, its genetic status is unclear.

In Fig. 4 are shown the results of experiments in which were determined the relative resistances of wild-type and resistant strains carrying the dominantly expressed mutations. Alleles $A H R I-11, A H R I-12$ and $A H R I-14$ consistently yielded higher resistance than $A H R I-9$ 
Table 1. Reversal of BAH toxicity by other amino acids

Cultures of strain $\mathrm{S} 288 \mathrm{C}$ were inoculated to an $\mathrm{OD}_{520}$ of about 0.03 and incubated as described in Methods. SD75 medium containing BAH and amino acids at the given concentrations was used. The results are the means of duplicates that differed by less than $10 \%$.

$\begin{array}{cccccc}\begin{array}{c}\text { BAH } \\ \text { concn } \\ (\mathrm{mM})\end{array} & \begin{array}{c}\text { Amino acid } \\ \text { concn } \\ (\mathrm{mM})\end{array} & \overbrace{\text { L-Asn }} & \text { L-Gln } & \text { L-Cit } & \text { D-Asn } \\ 0 & 0 & 1.6 & 1.6 & 1.6 & 1.6 \\ & 2 & 1.7 & 1.8 & 1.6 & 1.3 \\ & 10 & 1.8 & 1.8 & 1.6 & 1.3 \\ 1 & 0 & 0.06 & 0.06 & 0.06 & 0.06 \\ & 2 & 0.8 & 0.4 & 0.1 & 0.1 \\ & 10 & 1.5 & 1.4 & 0.1 & 0.1\end{array}$

or $A H R I-10$. The mutation from CAHR 13 engendered resistance relative to growth in medium without analogue about the same as $A H R I-9$ and $A H R I-10$, but strain CAHR13 consistently grew more slowly in unsupplemented media than the other strains.

\section{Asparaginase activities}

To confirm that resistance to BAH in these mutants was not associated with loss of activity of L-asparaginase I or asparaginase II, these enzymes were assayed in S288C, XE59-8B, and haploid strains carrying the resistance alleles. L-Asparaginase I was detected in all strains, averaging $9 \pm 2(\mathrm{SE}, n=8) \mathrm{nmol}$ ammonia $\min ^{-1}(\mathrm{mg} \text { protein })^{-1}$. Asparaginase II was assayed in stationary phase cells derepressed by nitrogen starvation (Dunlop \& Roon, 1975) and was present in all strains, averaging $239 \pm 8(\mathrm{SE}, n=8) \mathrm{nmol} \mathrm{ammonia} \min ^{-1}(\mathrm{mg} \text { protein })^{-1}$. Thus dominant resistance to $\mathrm{BAH}$ in these strains was not dependent upon loss of ability to synthesize either of these enzymes.

\section{Responses to hydroxylamine}

It is possible that these mutants were resistant to hydroxylamine that might contaminate BAH stocks, or that might have been released chemically or enzymically during selection and testing. However, when the ability of these strains to grow in the presence of hydroxylamine was tested, the mutants were not more resistant than wild-type cells. In fact, at a concentration of hydroxylamine that inhibited growth of wild-type cells by $20 \%(0.5 \mathrm{mM})$ or $80 \%(2.0 \mathrm{mM})$, the BAH-resistant mutants were perhaps slightly more sensitive to the amine than were wild-type cells. Thus, resistance appears to involve a response to the analogue itself rather than to hydroxylamine.

\section{Reversal of $B A H$ inhibition by amino acids}

If BAH exerts its inhibitory effects by altering asparagine metabolism in cells, then growth inhibition by the analogue might be prevented by including L-asparagine or related compounds in the growth medium. To test this possibility, cells of strain S288C were cultured in SD75 medium containing $1.0 \mathrm{~mm}-\mathrm{BAH}$ along with other individual amino acids at a concentration of $2.0 \mathrm{mM}$ or $10.0 \mathrm{mM}$. L-Asparagine and, to a lesser extent, L-glutamine considerably reduced the effects of the analogue (Table 1). The other 20 amino acids tested (including L-aspartate, Lglutamate, and D-asparagine) were much less effective. The results in Table 1 also show that only at a concentration of $10 \mathrm{mM}$ did L-asparagine fully reverse the inhibition by $1.0 \mathrm{mM}-\mathrm{BAH}$. It seems likely that BAH affects cells by altering some aspect of metabolism that involves (directly or indirectly) both L-asparagine and L-glutamine rather than affecting asparagine metabolism alone.

\section{Cross-resistance to $\gamma$-L-glutamylhydroxamate $(G G H)$}

The observation that L-glutamine substantially reduced inhibition of growth by BAH suggested that resistance to BAH might also affect glutamine metabolism. The results 


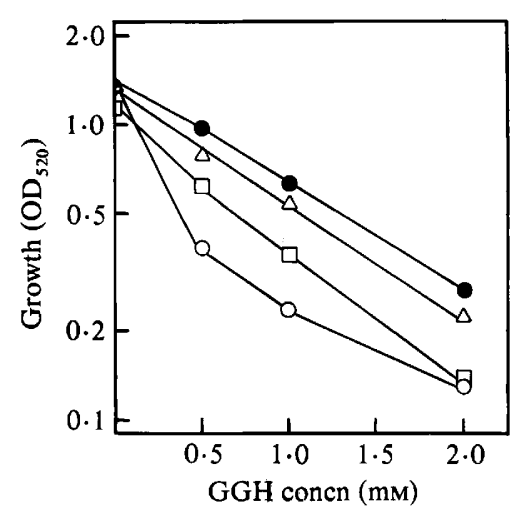

Fig. 5. Growth of S288C and its BAH-resistant derivatives in SD75 medium containing the indicated concentrations of GGH under the conditions described in Fig. 1. O, S288C; O, CAHR9, CAHR10, CAHR12, CAHR14; $\triangle$, CAHR11; $\square$, CAHR13.

summarized in Fig. 5 confirm this possibility. The BAH-resistant mutants also were partially resistant to the equivalent glutamine analogue GGH. Taken together, these results indicate that dominantly expressed alteration in this gene affects a metabolic function(s) that includes both Lasparagine and L-glutamine. Such effects might occur in other organisms, too. BAH-resistant mutants of $A$. nidulans also were resistant to GGH (Kinghorn \& Pateman, 1977).

\section{L-Asparagine synthetase activity}

Activity of L-asparagine synthetase was assayed in extracts of the BAH-sensitive strain

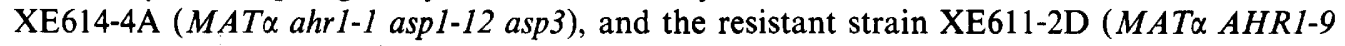
asp1-12 asp3). Strains deficient in both asparaginase activities were used to avoid possible interference with the assay by these enzymes. Activities in the strains were not significantly different. In XE614-4A the activity was $5 \cdot 1 \pm 0.3 \mathrm{nmol} \mathrm{L}$-asparagine $\mathrm{min}^{-1}$ (mg protein) ${ }^{-1}$, while in $\mathrm{XE} 611-2 \mathrm{D}$ it was $5 \cdot 6 \pm 1 \cdot 0 \mathrm{nmol} \mathrm{L}$-asparagine $\min ^{-1}$ (mg protein $)^{-1}$. S. cerevisiae possesses two L-asparagine synthetases (Ramos \& Wiame, 1980), neither of which appears to have been overly active in the mutant.

\section{Amino acid transport}

One important way in which $S$. cerevisiae regulates amino acid metabolism is by altering the rate at which exogenous amino acids enter the cell (extensively reviewed by Cooper, 1982). Both general and specific amino acid transport systems exist in this species, and their regulation is complex. In many studies in this field, mutants resistant to amino acid analogues were used to elucidate the mechanisms by which the metabolism of individual amino acids is controlled. For several amino acids, at least a part of that control is at the level of amino acid transport: strains resistant to an analogue often have reduced ability to take up the cognate amino acid (Cooper, 1982). Thus, we determined whether our BAH-resistant mutants were affected in transport of Lasparagine.

As shown in Table 2, when cells were grown in medium containing ammonium ion as the sole nitrogen source, the $A H R 1-9$ mutation strongly reduced the ability to take up L-asparagine from the surrounding medium. Similar results were obtained when $0.02 \mathrm{mM}$-substrate was present except that rate of transport in the wild-type diploid (XE521) was reduced to approximately $10 \mathrm{nmol} \mathrm{L}$-asparagine $\min ^{-1}(\mathrm{mg} \text { protein })^{-1}$. In these mutants, then, a metabolic function that affects the transport of L-asparagine has been altered.

Subsequent experiments showed that transport of other amino acids also was reduced in BAH-resistant mutants growing in a medium containing ammonium ion. As shown in Table 2, transport of L-aspartate, L-proline, and L-citrulline was reduced in the mutants under these conditions. Numerous experiments with both haploid and diploid cells carrying various $A H R I$ mutations and that from CAHR13 confirmed these conclusions. 
Table 2. Initial rates of transport of amino acids by diploid cells grown in SD20 medium

Transport rates were determined as described in Methods with $0.1 \mathrm{~mm}$-amino acid. Results are from a typical experiment; experiments were repeated several times, and rates were always reproducible to within about $15 \%$ among experiments.

Strain

$\begin{array}{ll} & \text { Genotype } \\ & \\ +/+ & +/+ \\ +/+ & +/ A H R I-9 \\ +/+ & \text { AHRI-9/AHRI-9 } \\ \text { gapl/gapl } & +/+ \\ \text { gapl/gapl } & +\mid \text { AHRl-9 } \\ \text { gapl/gapl } & \text { AHRI-9/AHRI-9 }\end{array}$

XE521

XE597

XE598

XE596

XE609

$\mathrm{XE} 610$

\begin{tabular}{|c|c|c|c|}
\hline \multicolumn{4}{|c|}{ 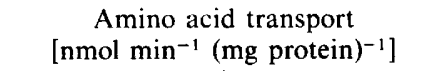 } \\
\hline L-Cit & L-Asn & L-Asp & L-Pro \\
\hline 62 & 37 & 59 & 7 \\
\hline$<1$ & $<1$ & 3 & $<1$ \\
\hline$<1$ & $<1$ & 7 & $<1$ \\
\hline$<1$ & 2 & 10 & 2 \\
\hline$<1$ & $<1$ & 8 & $<1$ \\
\hline$<1$ & $<1$ & 9 & $<1$ \\
\hline
\end{tabular}

The reduction in transport ability by mutation in the $A H R I$ gene is dominantly expressed (Table 2) but is not unconditional. When cells were grown in medium containing urea or Lproline as a nitrogen source, transport activity was present, although at reduced levels in all strains (results not shown).

As stated above, most amino acids can enter yeast cells by transport systems specific for that amino acid or for a closely related group of amino acids. In addition, a major system exists by which many amino acids can enter, the general amino acid permease (GAP), which has been the subject of extensive study (see, for example, Grenson, 1983; Courchesne \& Magasanik, 1983). One distinguishing feature of the GAP system is that it is the sole route by which L-citrulline enters the cell; L-proline, L-aspartate, and L-asparagine also are substrates for this function. Activity of this permease is affected by a number of genes (Cooper, 1982; Courchesne \& Magasanik, 1983; Grenson, 1983), one of which is GAPI. Mutation in this gene eliminates transport of substrates of the GAP. As shown in Table 2, diploid strains homozygous for the gapl mutation did not transport L-citrulline and exhibited strongly reduced transport of the other amino acids tested. This phenotype was recessive, as expected for the gapl mutation; GAP1/gapl heterozygotes had full transport activity (results not shown). Lack of transport in GAP-deficient strains was unconditional, in contrast with BAH-resistant mutants. The latter, for example, grow well in medium in which L-citrulline is the only nitrogen source; GAPdeficient mutants do not.

Genetic crosses clearly showed that $a h r l$ and gapl assort independently. Additionally, ahrl assorted independently of the aap gene, mutation in which is known to affect the GAP function as well as many specific amino acid transport systems (Cooper, 1982). (Indeed, recessive BAH resistance mutations carried by strains CAHR 3 and CAHR7 mentioned earlier proved to be allelic with the $A A P$ gene.)

The dominantly expressed mutations in the $a h r l$ gene appear to effect resistance to BAH by making the GAP function sensitive to ammonium ion in the growth medium. Several genes have been described that affect the sensitivity of this function to ammonium ion (Cooper, 1982; Courchesne \& Magasanik, 1983; Grenson, 1983), including the amc gene in strains derived from S288C (Rytka, 1975). Mutation from $a m c^{-}$to $a m c^{+}$renders the GAP function dominantly sensitive to ammonium ion. It is possible that the $a h r l$ gene is allelic with amc; we have been unable to derive and authenticate a known amc mutation. However, two observations suggest that loss of GAP function alone, which was the only function addressed by Rytka (1975), probably does not solely account for BAH resistance in these strains.

First, strains bearing the gapl mutation and deficient in GAP were not highly resistant to BAH (Fig. 3). Indeed, they were only marginally more resistant than wild-type cells. Second, as shown in Table 1, L-citrulline in the growth medium failed to reverse the toxic effects of BAH as might have been expected if both amino acids were taken up by the same transport system. Even when L-citrulline $(10 \mathrm{mM})$ was the only source of nitrogen in the growth medium and should have 
Table 3. Inhibition of L-asparagine transport by BAH and L-citrulline in cells of strain S288C grown in SD20 medium

The initial rate of transport of L-asparagine at the indicated concentrations was measured in the presence of BAH $(0.5 \mathrm{~mm})$ or L-citrulline $(0.5 \mathrm{~mm})$. Rates are given for a typical experiment and were reproducible to within about $15 \%$ among experiments.

\begin{tabular}{|c|c|c|c|c|}
\hline \multirow{2}{*}{$\begin{array}{l}\text { L-Asn } \\
\text { concn } \\
(\mathrm{mM})\end{array}$} & \multirow[b]{2}{*}{ Inhibitor } & \multicolumn{3}{|c|}{ 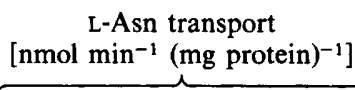 } \\
\hline & & None & BAH & L-Cit \\
\hline $\begin{array}{l}0.05 \\
0.10 \\
0.50\end{array}$ & & $\begin{array}{r}47 \\
68 \\
110\end{array}$ & $\begin{array}{l}26 \\
43 \\
98\end{array}$ & $\begin{array}{r}5 \\
12 \\
49\end{array}$ \\
\hline
\end{tabular}

strongly reduced GAP transport availability for other substrates, $1 \mathrm{~mm}$-BAH was fully toxic (results not shown). In contrast, L-asparagine in the growth medium greatly reduced BAH toxicity (Table 1).

It is possible that L-asparagine and BAH use, for entry into cells, different sites on the GAP than L-citrulline, and that both citrulline and asparagine carriers are affected in BAH-resistant mutants. However, this is unlikely. L-Citrulline strongly reduced the uptake of L-asparagine and, indeed, was a better competitor against $L$-asparagine than was the asparagine analogue (Table 3).

It seems more likely that dominant resistance to $\mathrm{BAH}$ is the result of more subtle alterations in the regulation of asparagine metabolism in these cells, and, since the mutants are cross resistant to GGH, it is possible that more fundamental aspects of overall nitrogen metabolism are altered in them. For example, it is possible that these mutations pleiotropically affect the GAP function and the poorly characterized L-asparagine/L-glutamine specific transport mechanism (Cooper, 1982).

The goal of this project has not been to provide a detailed study of amino acid transport or nitrogen metabolism in yeast. We have shown that mutations in several genes confer resistance to BAH and that neither loss of asparaginase activity nor excessive synthesis of L-asparagine need be involved. The observations presented here strongly suggest that a study of resistance to BAH might prove fruitful in laboratories devoted to this field. In particular, mutants such as those described here might provide valuable information about the relationship between Lasparagine and L-glutamine transport and utilization in this species.

I thank C. Boyd for excellent technical assistance and R. K. Dawe for isolating the strains derived from XE22720A. This work was supported by the Agricultural Experiment Station, University of California, Riverside, USA.

\section{REFERENCES}

ANDRulis, I. L. \& Siminovitch, L. (1982). Isolation and characterization of Chinese hamster ovary cell mutants resistant to the amino acid analog betaaspartylhydroxamate. Somatic Cell Genetics 8, 533545.

ARST, H. N. JR \& Bailey, C. R. (1980). Genetic evidence for a second asparaginase in Aspergillus nidulans. Journal of General Microbiology 121, 243247.

BRADFORD, M. M. (1976). A rapid and sensitive method for the quantification of microgram quantities of protein utilizing the principle of protein-dye binding. Analytical Biochemistry 72, 248-254.

COOPER, T. G. (1982). Transport in Saccharomyces cererisiae: metabolism and gene expression. In The Molecular Biology of the Yeast Saccharomyces: Metabolism and Gene Expression, pp. 399-461. Edited by J. N. Strathern, E. W. Jones \& J. R.
Broach. Cold Spring Harbor, New York: Cold Spring Harbor Laboratory.

Courchesne, W. E. \& Magasanik, B. (1983). Ammonia regulation of amino acid permeases in Saccharomyces cerevisiae. Molecular and Cellular Biology 3, 672-683.

Drainas, C., Kinghorn, J. R. \& Pateman, J. A. (1977). Aspartic hydroxamate resistance and asparaginase regulation in the fungus Aspergillus nidulans. Journal of General Microbiology 98, 493-501.

DunloP, P. C. \& Roon, R. J. (1975). L-Asparaginase of Saccharomyces cerevisiae: an extracellular enzyme. Journal of Bacteriology 122, 1017-1024.

Gantt, J. S., Chiang, C.-S., Hatfield, G. W. \& ARFIN, S. M. (1980). Chinese hamster ovary cells resistant to beta-aspartylhydroxamate contain increased levels of asparagine synthetase. Journal of Biological Chemistry 255, 4808-4813. 
GRENSON, M. (1983). Inactivation-reactivation process and repression of permease formation regulate several ammonia-sensitive permeases in the yeast Saccharomyces cerevisiae. European Journal of Biochemistry 133, 135-139.

Jones, G. E. \& MoRTimer, R. K. (1970). L-Asparaginase-deficient mutants of yeast. Science 167, 181-182.

Jones, G. E. \& Mortimer, R. K. (1973). Biochemical properties of yeast L-asparaginase. Biochemical Genetics 9, 131-146.

KIM, K. W. \& Roon, R. J. (1984). Asparaginase II of Saccharomyces cerevisiae: positive selection of two mutations that prevent enzyme synthesis. Journal of Bacteriology 157, 958-961.

Kinghorn, J. R. \& Pateman, J. A. (1977). Nitrogen metabolism. In Genetics and Physiology of Aspergillus, pp. 147-202. Edited by J. E. Smith \& J. A. Pateman. London: Academic Press.

Lasko, P. F. \& Brandriss, M. C. (1981). Proline transport in Saccharomyces cerevisiae. Journal of Bacteriology 148, 241-247.
Mortimer, R. K. \& Hawthorne, D. C. (1969). Yeast genetics. In The Yeasts, vol. 1, pp. 385-460. Edited by A. H. Rose \& J. S. Harrison. New York: Academic Press.

Pauling, K. D. \& Jones, G. E. (1980). Asparaginase II of Saccharomyces cerevisiae: dynamics of accumulation and loss in rapidly growing cells. Journal of General Microbiology 117, 423-430.

Ramos, F. \& WIAME, J.-M. (1979). Synthesis and activation of asparagine in asparagine auxotrophs of Saccharomyces cerevisiae. European Journal of Biochemistry 94, 409-417.

Ramos, F. \& Wiame, J.-M. (1980). Two asparagine synthetases in Saccharomyces cerevisiae. European Journal of Biochemistry 108, 373-377.

RytKa, J. (1975). Positive selection of general amino acid permease mutants in Saccharomyces cerevisiae. Journal of Bacteriology 121, 562-570.

Sherman, F., Fink, G. R. \& Lawrence, C. W. (1971). Methods in Yeast Genetics. Cold Spring Harbor, New York: Cold Spring Harbor Laboratory. 